\title{
Pressure-Dependent Relaxation in the Photoexcited Mott Insulator ET-F 2 TCNQ: Influence of Hopping and Correlations on Quasiparticle Recombination Rates
}

\author{
M. Mitrano, ${ }^{1, *}$ G. Cotugno, ${ }^{1,2}$ S. R. Clark, ${ }^{3,2}$ R. Singla, ${ }^{1}$ S. Kaiser,,${ }^{1,}$ J. Stähler, ${ }^{4}$ R. Beyer, ${ }^{5}$ M. Dressel, ${ }^{5}$ L. Baldassarre, ${ }^{6}$ \\ D. Nicoletti, ${ }^{1}$ A. Perucchi, ${ }^{7}$ T. Hasegawa, ${ }^{8}$ H. Okamoto, ${ }^{9}$ D. Jaksch, ${ }^{2,3}$ and A. Cavalleri ${ }^{1,2, \$}$ \\ ${ }^{1}$ Max Planck Institute for the Structure and Dynamics of Matter, Luruper Chaussee 149, 22761 Hamburg, Germany \\ ${ }^{2}$ Department of Physics, Oxford University, Clarendon Laboratory, Parks Road, OX1 3PU Oxford, United Kingdom \\ ${ }^{3}$ Centre for Quantum Technologies, National University of Singapore, 3 Science Drive 2, Singapore 117543, Singapore \\ ${ }^{4}$ Fritz. Haber Institute of the Max Planck Society, Faradayweg 4-6, 14195 Berlin, Germany \\ ${ }^{5}$ 1. Physikalisches Insitut, Universität Stuttgart, Pfaffenwaldring 57, 70550 Stuttgart, Germany \\ ${ }^{6}$ Center for Life NanoScience@Sapienza, Istituto Italiano di Tecnologia, V.le Regina Elena 291, 00185 Rome, Italy \\ ${ }^{7}$ INSTM UdR Trieste-ST and Elettra - Sincrotrone Trieste S.C.p.A., S.S. 14 km 163.5 in Area Science Park, \\ 34012 Basovizza, Trieste Italy \\ ${ }^{8}$ National Institute of Advanced Industrial Science and Technology (AIST), Tsukuba 305-8562, Japan \\ ${ }^{9}$ Department of Advanced Material Science, University of Tokyo, Chiba 277-8561, Japan
}

(Received 28 August 2013; published 18 March 2014)

\begin{abstract}
We measure the ultrafast recombination of photoexcited quasiparticles (holon-doublon pairs) in the one dimensional Mott insulator ET- $\mathrm{F}_{2}$ TCNQ as a function of external pressure, which is used to tune the electronic structure. At each pressure value, we first fit the static optical properties and extract the electronic bandwidth $t$ and the intersite correlation energy $V$. We then measure the recombination times as a function of pressure, and we correlate them with the corresponding microscopic parameters. We find that the recombination times scale differently than for metals and semiconductors. A fit to our data based on the time-dependent extended Hubbard Hamiltonian suggests that the competition between local recombination and delocalization of the Mott-Hubbard exciton dictates the efficiency of the recombination.
\end{abstract}

DOI: 10.1103/PhysRevLett.112.117801

The recombination of hot carriers in solids is a fundamental process of interest to nonlinear optics and to device applications, as well as a spectroscopic tool that exposes the physics of interacting microscopic degrees of freedom. "Hot electron" spectroscopy has been applied extensively to metals and semiconductors, for which well-established models have been developed.

For direct gap semiconductors recombination occurs at a rate that depends on the joint density of states between valence and conduction bands $\propto\left(\partial E_{v} / \partial k\right)\left(\partial E_{c} / \partial k\right)$, and is thus expected to slow down with the square of the bandwidth $\tau \propto t^{2}$. On the other hand, in the case of metals, the dynamics are well captured by the two-temperature model $[1,2]$, which considers the energy stored in the optically excited nonequilibrium electron distribution as flowing into the lattice at a rate determined by the electronphonon coupling strength and by the electronic and lattice heat capacities. As the relaxation of hot electrons accelerates with smaller electronic specific heat, and because $c_{v}^{e}$ is proportional to the density of states at the Fermi level [3], for metals relaxation should accelerate linearly with the reciprocal of the bandwidth $\tau \propto 1 / t$.

For solids with strongly correlated electrons, the dependence of nonequilibrium quasiparticle recombination rates on the microscopic parameters has not been systematically
PACS numbers: 78.30.Jw, 78.20.Ci, 78.47.jg, 78.55.Kz

investigated and it is not well understood. In this Letter, we study the recombination of impulsively excited quasiparticles in a one dimensional Mott insulator, in which we tune electronic bandwidth and intersite correlation energy by applying external pressure. We find that the recombination of quasiparticles accelerates for increasing bandwidth, as expected for a metal, but with a dependence on microscopic parameters that is unique to the physics of electronic insulators in one dimension and that descends from a competition between local decay and coherent delocalization of photoexcited holon-doublon pairs [4].

We study bis-(ethylendithyo)-tetrathiafulvalenedifluorotetracyano-quinodimethane (ET- $\left.\mathrm{F}_{2} \mathrm{TCNQ}\right)$, a half filled organic salt with quasi-1D electronic structure, negligible electron-phonon interaction [5], and with electronic properties that are well captured by a $1 \mathrm{D}$ extended Hubbard model $[4,6]$

$H=-t \sum_{j \sigma}\left(\hat{c}_{j \sigma}^{+} \hat{c}_{j+1 \sigma}+\right.$ H.c. $)+V \sum_{j} \hat{n}_{j} \hat{n}_{j+1}+U \sum_{j} \hat{n}_{j \uparrow} \hat{n}_{j \downarrow}$.

Our experiments were performed under external pressure, which reduces the lattice spacing between the molecular sites and tunes the hopping amplitude $(t)$ and repulsion 
(attraction) between electron (electron-hole) pairs on neighboring sites $(V)$. In the simplified case of hydrogenic wave functions in one dimension [7,8], $t$ depends exponentially on the lattice spacing $R$ as $t(R) \propto(1+\alpha R) e^{-\alpha R}$. The decrease in the lattice spacing $R$ results then in an increase of the intersite Coulomb repulsion $V$ as $V(R) \propto e^{2} / R$ [9]. Within these simplifying assumptions, the onsite Coulomb interaction $U$, which is determined by the local electronic properties of the molecular sites, is considered independent on the lattice spacing [10].

For calibration, we first measured and fitted the static optical properties as a function of pressure (see Supplemental Material [11]). In Fig. 1, we report the reflectivity measured at $300 \mathrm{~K}$ for electric field polarized along the 1D charge transfer direction ( $a$ axis). The corresponding optical conductivity $\sigma_{1}(\omega)$ was extracted by a Kramers-Kronig consistent fit. At ambient conditions [see Figs. 1(a) and (b)], $\sigma_{1}(\omega)$ exhibits a prominent peak near $700 \mathrm{meV}$, indicative of electron-electron correlations and of a Mott gap [5]. As pressure was applied, the $700 \mathrm{meV}$ charge transfer (CT) resonance was observed to shift to the red at a rate of $70 \mathrm{meV} / \mathrm{GPa}$, broadening toward high frequencies [see Figs. 1(c) and (d)]. This pressure dependence is consistent with the behavior measured previously in other quasi-1D compounds $[12,13]$. For all measured photon energies $(>75 \mathrm{meV})$, no Drude response was observed at any pressure (0-2 GPa), indicating that the material remains insulating and one dimensional $[13,14]$. The vibrational peaks at frequencies below $400 \mathrm{meV}$ exhibited no pressure dependence, excluding significant intramolecular structural rearrangement.

The data were analyzed using a model of the optical conductivity based on the extended Hubbard Model of
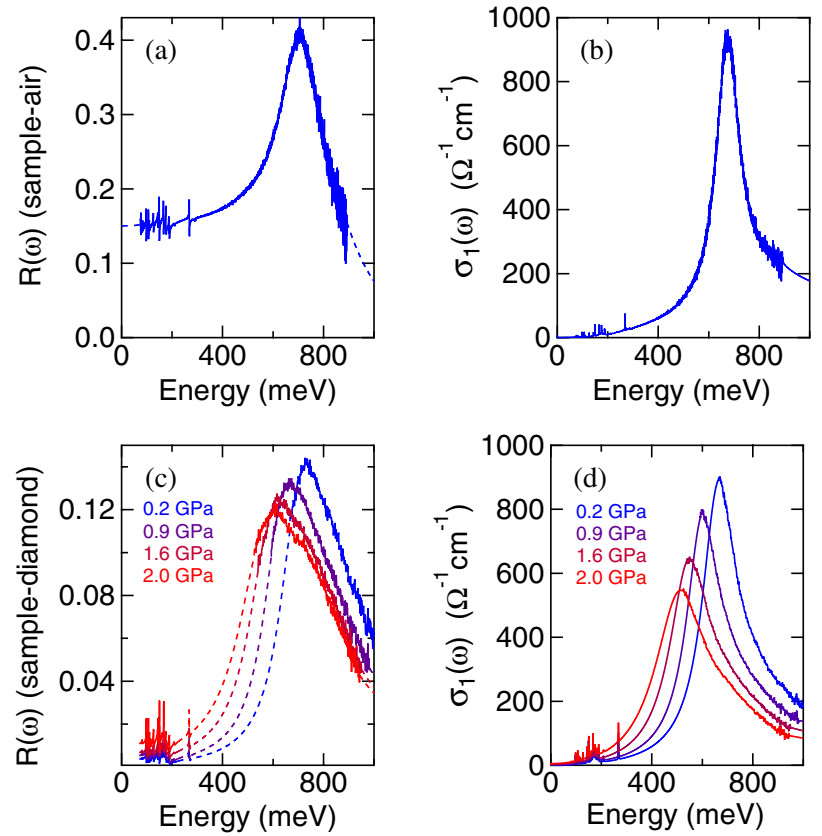

FIG. 1 (color online). (a) Static reflectivity at ambient pressure of the ET-F $\mathrm{F}_{2} \mathrm{TCNQ}$, measured with the electric field parallel to the $a$ axis. (b) Real part of the optical conductivity $\sigma_{1}(\omega)$ at ambient pressure. (c) Steady state reflectivity of the ET-F $\mathrm{F}_{2}$ TCNQ along the $a$ axis for selected pressures. (d) Pressure dependence of the optical conductivity $\sigma_{1}(\omega)$.

Eq. (1). The onsite repulsion $U$ was assumed to be a constant $845 \mathrm{meV}$ at all pressures. The large Mott gap of ET- $F_{2}$ TCNQ enables a $1 / U$ strong coupling expansion of the extended Hubbard model giving a reduced optical conductivity $\omega \sigma_{1}(\omega)$ in the form

$$
\omega \sigma_{1}(\omega)=g_{0} t^{2} e^{2}\left\{\Theta(V-2 t) \pi\left[1-\left(\frac{2 t}{V}\right)^{2}\right] \delta\left(\omega-\omega_{C T}\right)+\Theta(4 t-|\omega-U|) \frac{2 t \sqrt{1-\left[\frac{\omega-U}{4 t}\right]^{2}}}{V\left(\omega-\omega_{\mathrm{CT}}\right)}\right\}
$$

where $e$ is the electronic charge, $\Theta$ is the Heaviside function and $g_{0}=2.65$ is the zero-momentum form factor accounting for the spin degrees of freedom $[15,16]$ (see Supplemental Material [11]).

This analytical result highlights the two dominant contributions to $\omega \sigma_{1}(\omega)$ resulting from the relevant quasiparticles of this system. The first is a delta peak located at $\omega_{\mathrm{CT}}=U-V-4 t^{2} / V$, and corresponds to a MottHubbard exciton composed of a bound holon-doublon (HD) pair. A second broad peak is centered around $U$, with a bandwidth of $8 t$, and corresponds to the continuum of states associated with unbound particle-hole (PH) excitations $[15,16]$. These two types of excitations are visualized for the 1D lattice model in Fig. 2(d). As pressure is applied, the exciton peak shifts to the red, whereas the continuum remains centered at $U$ and broadens with the increase in bandwidth.

A comparison of the measured and fitted $\omega \sigma_{1}(\omega)$, normalized to the peak value of the spectrum measured at ambient pressure, is reported for three selected pressures in Figs. 2(a)-(c) as black (measured data) and gray (theory fits) curves. The fit has been performed here after subtraction of the low-frequency tail of the intramolecular absorption bands near $3.5 \mathrm{eV}[4,5]$. The fitted values for $t$ and $V$ are summarized in Fig. 2(e). The nearest neighbor interaction $V$ and the hopping amplitude $t$ are both observed to increase with pressure, from 120 to $203 \mathrm{meV}$ and from 40 to $85 \mathrm{meV}$, respectively. 


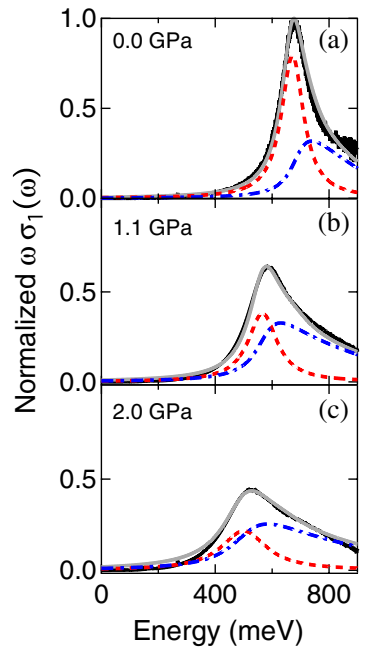

$$
\begin{gathered}
\$ \circ \$ \phi \phi \\
E_{H D}(P)=U-V(P)-4^{t^{2}(P)} \\
\$ \circ \phi \$ \phi
\end{gathered}
$$$$
E_{P H}(P)=U
$$

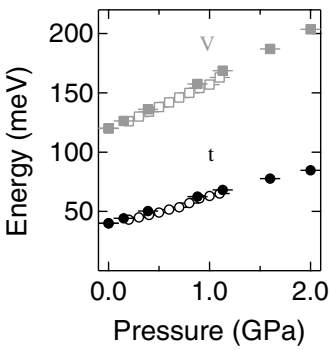

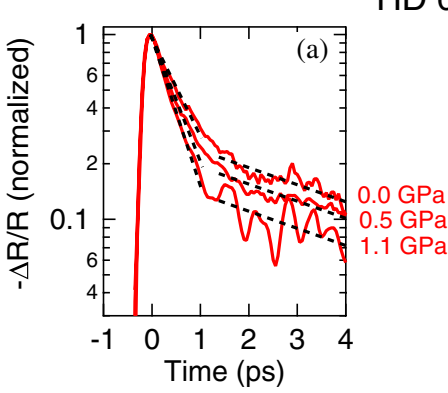

HD decay

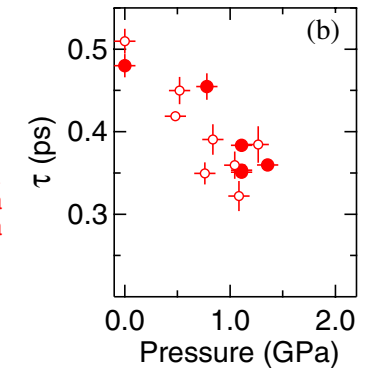

$\mathrm{PH}$ decay
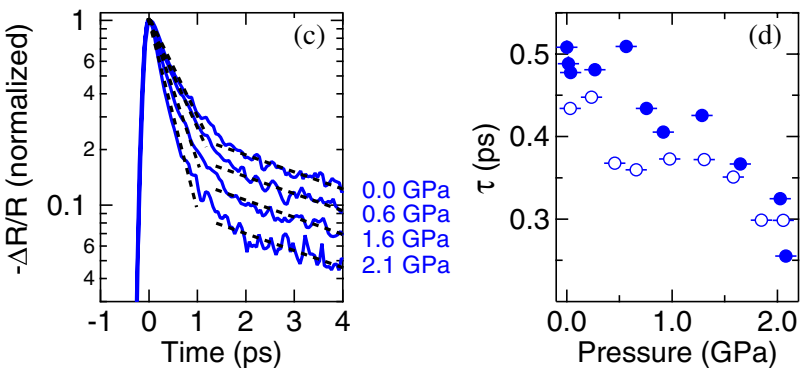

FIG. 3 (color online). (a) Normalized $\Delta R / R$ time domain curves on the holon-doublon pair peak (HD) for selected pressures (solid lines). Black dashed lines are guides to the eye showing the biexponential decay, linear on a log scale. (b) Holon-doublon recombination lifetimes extracted from a fit to the data. Filled and empty symbols identify distinct experimental runs. (c) Normalized $\Delta R / R$ time domain curves on the particlehole continuum peak $(\mathrm{PH})$ for selected pressures (solid lines). Black dashed lines are guides to the eyes showing the biexponential decay. (d) Particle-hole continuum recombination lifetimes extracted from a fit to the data. Filled and empty symbols identify distinct experimental runs.

to $350 \mathrm{fs}$ between 0 and $1.3 \mathrm{GPa}$ ) for bound holon-doublon pairs and $85 \mathrm{fs} / \mathrm{GPa}$ (470 to 300 fs between 0 and $2 \mathrm{GPa}$ ) for the particle hole continuum. Both holon-doublon and particlehole excitations exhibited similar recombination times and similar dependence on pressure. Indeed, from the static fitting discussed above, the ratio $V / t$ is reduced from $V / t \sim 3.0$ to $V / t \sim 2.4$, close to the critical ratio $V / t \sim 2$ for which the exciton peak cannot be isolated from the continuum $[15,16]$. The slow time constant of $2.5 \mathrm{ps}$, interpreted here as resulting from the thermalization of high-energy molecular modes heated by the recombination of quasiparticles, was independent on pressure.

To analyze this pressure dependence quantitatively, we first note that recombination of bound holon-doublon pairs (or disassociated pairs throughout the particle hole continuum) must involve dissipation of an excess energy of order $U-V$ by coupling to a bath. In the absence of such a bath the only possible decay paths involve kinetic energy transfer (creation of particle-hole pairs) or spin excitations within the Hubbard model itself, where the relevant energy scales are the bandwidth $t$ and the exchange coupling $t^{2} /(U-V)$, respectively [17]. For $U \gg V, t$ a large number of scattering events is required for 


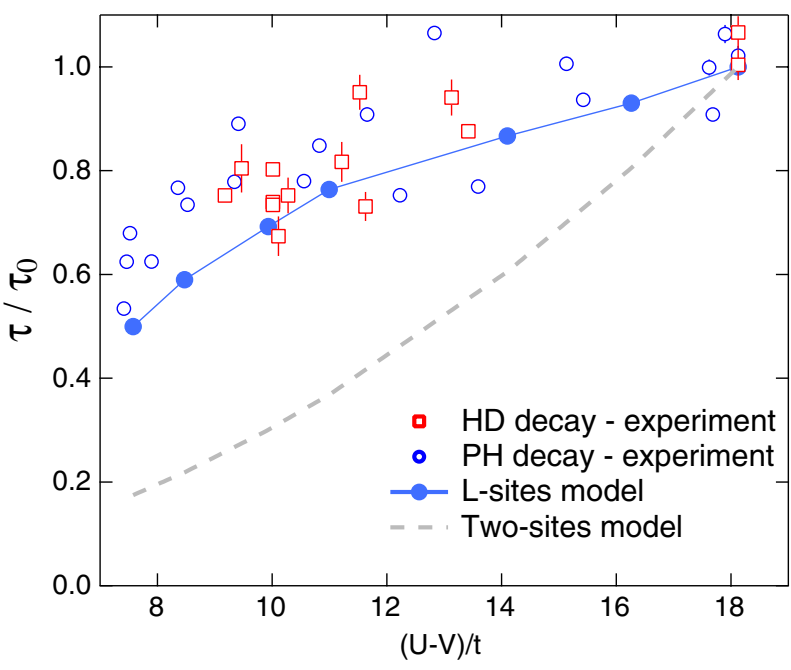

FIG. 4 (color online). Experimental relaxation times (empty symbols) for both HD and PH resonant excitation normalized to their ambient pressure values $\tau_{0}$ and shown as a function of $(U-V) / t$. The calculated holon-doublon lifetimes (filled circles) found from solving an $L$-site effective model (see Fig. 5) with spin-boson scaling for the bare decay rate. For comparison the $L=1$ dimer result is shown (dashed line).

recombination, leading to a decay rate that is exponentially small with $U / t$ [17-20]. Owing to the weak electronphonon coupling in ET- $\mathrm{F}_{2}$ TCNQ [5], and lack of efficient radiative emission, it is reasonable to assume that highfrequency molecular modes are the primary scattering partner for the rapid decay of hot quasiparticles observed in our experiment.

In the following discussion, we assume that recombination occurs locally and the density of holon-doublon excitations is sufficiently low that only a single pair need be considered. This is substantiated by the measured fluence dependence. In the range explored in our experiments the signal scaled linearly with the laser fluence, while recombination rates were found to be independent of it. This behavior suggests that excitations are sparsely distributed and recombination is localized.

The simplest possible model involves a dimer of ET molecules coupled to a continuum of bosonic modes, as is commonly used to model dissipative electron transfer $[21,22]$. The holon-doublon and singly occupied configurations of the dimer, with an energy gap $U-V$ and tunneling $t$ between them, comprise a two-level system of the spin-boson model [23]. We take a super-Ohmic form for spectral function $\mathcal{J}(\omega)$ appropriate for electron-phonon interactions [24]. Moreover, given $U-V \gg t$, the system is in the large-bias regime where the far-off-resonant tunneling mediates the decay of the holon-doublon pair by dissipating the large energy gap into the bosonic reservoir. The rate of decay $\Gamma$ in this limit is proportional to $t^{2} /(U-V)^{2} \mathcal{J}(U-V)$ [23]. As the dominant contributions to the spectral function at the energy gap $\mathcal{J}(U-V)$ arise from local vibrational modes it is assumed to be independent of pressure. This then gives a scaling of $\Gamma$ with the pressure dependent microscopic parameters as $t^{2} /(U-V)^{2}$, which sensibly predicts an increase with $t$ and a decrease with $U-V$.

In Fig. 4 the experimentally measured holon-doublon and particle-hole continuum decay rates, normalized to the ambient pressure decay rate $1 / \tau_{0}$, are plotted as a function of $(U-V) / t$. The dashed line shows the predicted change in the recombination rate (see Supplemental Material [11] for details), consisting of a more than fivefold increase at the largest pressure. Thus, the two-site model on its own does not reproduce the experimentally measured trend.

We note that previous time-resolved spectroscopy experiments showed that at least three sites are necessary to account for the photoresponse [4], owing to the possibility for bound holon-doublon pairs next to a singly occupied site $\mid \cdots, 0$ 年 $\ldots\rangle\rangle$ to tunnel into a configuration of the type $\left|\cdots 0_{1}-\cdots\right\rangle$, where $|\cdots 0 \cdots\rangle$ represents a holon, $|\cdots \uparrow, \cdots\rangle$ a doublon, and $|\cdots, \ldots\rangle$ represents a singly occupied site of either spin. This separation occurs at a rate determined by the hopping amplitude $t$ and is limited by a barrier $V$, where both of these increase with pressure. Importantly, the configuration $\left|\cdots_{-} 0_{-} \hat{1}_{-} \ldots\right\rangle$ has a lower probability to recombine than the configuration $|\cdots, 01 \ldots\rangle$, as two hopping events are necessary.

To include this effect, we employed an effective model based on the sketch of Fig. 5, derivable from the strongcoupling limit (see Supplemental Material [11]), where in addition to the singly-occupied configuration $|g\rangle$ and adjacent holon-doublon pair $|0\rangle$, other states $|l\rangle$, representing holon and doublons separated by $l$ sites were considered, up to maximum distance $L$. The potential energy of the model then

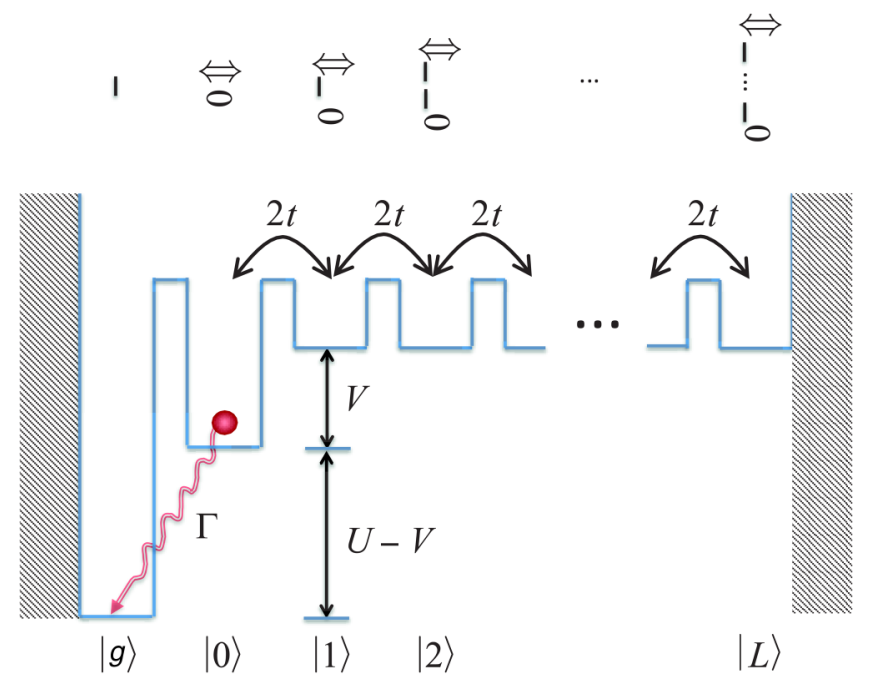

FIG. 5 (color online). Effective model describing holondoublon dynamics in the strong-coupling limit. The state $|g\rangle$ is the ground state containing no holons or doublons, whereas the state $|0\rangle$ represents an adjacent (zero separation) holon-doublon pair. The remaining states $|l\rangle$ represent the holon and doublon being separated by $l$ sites. In the limit $L \rightarrow \infty$ these unbound states form the particle-hole continuum. The relaxation to $|g\rangle$ at a bare spin-boson rate $\Gamma$ only occurs locally from state $|0\rangle$. 
mimicked the interactions of the equivalent many-body configurations in the full, extended Hubbard model. Importantly, the optical conductivity of this effective model, when $L \rightarrow \infty$, exactly reproduces that of Eq. (2) for the extended Hubbard model (see Supplemental Material [11]).

Local recombination was added to this effective model via a Markovian quantum dissipation process, appropriate for the super-Ohmic large-bias limit [25] (see Supplemental Material [11]), that incoherently drives only the transition $|0\rangle \rightarrow|g\rangle$ at a bare rate $\Gamma$. This effective model reveals that even the addition of one ionized holon-doublon state $|1\rangle$ which is unaffected by the decay, i.e., taking $L=1$, causes the suppression of the actual decay rate $\Gamma_{\text {eff }}$ to $|g\rangle$ from the bare rate $\Gamma$ as

$$
\Gamma_{\mathrm{eff}}=\frac{\Gamma}{2}\left(1+\frac{V}{\sqrt{V^{2}+16 t^{2}}}\right) .
$$

We applied this effective model by first fitting the bare rate $\Gamma$ to reproduce the observed zero pressure decay rate. For all other pressures $\Gamma$ was increased according to the spin-boson scaling by using the values for $t, V$ reported in Fig. 2(e). The effective decay rate $\Gamma_{\text {eff }}$ was then calculated numerically from this model for different sizes $L$. As $L$ increases the suppression of $\Gamma_{\text {eff }}$ becomes more pronounced since the disassociated holon-doublon pair can ballistically separate to larger distances remaining immune to the local decay. Thus, we find that coherent dynamics on more than two sites, accounting for the break-up of the holon-doublon pair, competes and slows down the recombination caused by the local decay process.

In conclusion, we have experimentally investigated the pressure dependence of hot quasiparticle recombination in a one dimensional Mott insulator. By fitting the steady state infrared properties with a model based on the extended Hubbard Hamiltonian, we extracted the pressure dependence of theHubbard parameters $t$ and $V$ up to $2.0 \mathrm{GPa}$, and correlated them to the recombination rates. A key inference made by comparing the experimentally determined dependence to theory is that the decay of quasiparticles is likely connected to the coherent evolution of holon-doublon pairs immediately after excitation. Based on this idea, it may be possible in the future to accelerate or decelerate the photoinduced dynamics of correlated electron systems by pulse shaping and by coherent optical control techniques. Such ability would have interesting ramifications in both fundamental science and applications.

We thank P. Di Pietro and A. Dengl for technical support in the equilibrium optical measurements and M. Eckstein for fruitful discussions. This research has been funded by the European Research Council under the European Union's Seventh Framework Programme (FP7/20072013)/ERC Grant Agreement No. 319286. D. J. and S. R. C. thank the National Research Foundation and the Ministry of Education of Singapore for support.
Matteo.Mitrano@mpsd.mpg.de

†Stefan.Kaiser@mpsd.mpg.de

*Andrea.Cavalleri@mpsd.mpg.de

[1] P. B. Allen, Phys. Rev. Lett. 59, 1460 (1987).

[2] S. I. Anisimov, B. L. Kapeliovich, and T. L. Perel'man, Sov. Phys. JETP 39, 375 (1974).

[3] N. W. Ashcroft and N. D. Mermin, Solid State Physics (Thomson Learning, London, UK, 1976).

[4] S. Wall, S. Wall, D. Brida, S. R. Clark, H. P. Ehrke, D. Jaksch, A. Ardavan, S. Bonora, H. Uemura, Y. Takahashi, T. Hasegawa et al. Nat. Phys. 7, 114 (2011).

[5] T. Hasegawa, S. Kagoshima, T. Mochida, S. Sugiura, and Y. Iwasa, Solid State Commun. 103, 489 (1997).

[6] H. Okamoto, H. Matsuzaki, T. Wakabayashi, Y. Takahashi, and T. Hasegawa, Phys. Rev. Lett. 98, 037401 (2007).

[7] E. P. Wohlfarth, Proc. Phys. Soc. London Sect. A 66, 889 (1953).

[8] N. F. Mott, Metal-Insulator Transitions (Taylor \& Francis, London, 1990), 2nd ed.

[9] G. D. Mahan, Many-Particle Physics (Plenum Press, New York, 1990), 2nd ed.

[10] L. I. Schiff, Quantum Mechanics (McGraw-Hill, New York, 1968), 3rd ed.

[11] See Supplemental Material at http://link.aps.org/ supplemental/10.1103/PhysRevLett.112.117801 for details about the determination of the equilibrium optical properties and for an extended discussion of the theoretical modeling developed throughout the paper.

[12] A. Pashkin, M. Dressel, and C. A. Kuntscher, Phys. Rev. B 74, 165118 (2006).

[13] A. Pashkin, M. Dressel, M. Hanfland, and C. A. Kuntscher, Phys. Rev. B 81, 125109 (2010).

[14] Also far infrared (37-80 meV) transmittance data in the $a b$ plane (not shown) exclude the development of a transverse Drude-like delocalization and a consequent dimensional crossover within the investigated pressure range.

[15] F. H. L. Essler, F. Gebhard, and E. Jeckelmann, Phys. Rev. B 64, 125119 (2001).

[16] E. Jeckelmann, Phys. Rev. B 67, 075106 (2003).

[17] R. Sensarma, D. Pekker, E. Altman, E. Demler, N. Strohmaier, D. Greif, R. Jördens, L. Tarruell, H. Moritz, and T. Esslinger, Phys. Rev. B 82, 224302 (2010).

[18] M. Eckstein and P. Werner, Phys. Rev. B 84, 035122 (2011).

[19] N. Strohmaier, D. Greif, R. Jördens, L. Tarruell, H. Moritz, T. Esslinger, R. Sensarma, D. Pekker, E. Altman, and E. Demler, Phys. Rev. Lett. 104, 080401 (2010).

[20] Z. Lenarčič and P. Prelovsek, Phys. Rev. Lett. 111, 016401 (2013).

[21] R. Egger, C. H. Mak, and U. Weiss, J. Chem. Phys. 100, 2651 (1994).

[22] S. Tornow, R. Bulla, F. B. Anders, and A. Nitzan, Phys. Rev. B 78, 035434 (2008).

[23] A. Leggett, S. Chakravarty, A. Dorsey, M. Fisher, A. Garg, and W. Zwerger, Rev. Mod. Phys. 59, 1 (1987).

[24] U. Weiss, Quantum Dissipative Systems (World Scientific, Singapore, 2008), 3rd ed.

[25] D. P. S. McCutcheon and A. Nazir, Phys. Rev. B 83, 165101 (2011). 